\section{The Mystery of the Turin Shroud} Walter C. McCrone, McCrone Associates

In 1355, or close thereto, a 3-foot wide and 14-foot long linen cloth suddenly appeared in history. Greeted rapturously by millions of Christians, it has maintained its acceptance as the true Shroud of Christ from 1300 years earlier. In 1978, a group of about 30 U.S. scientists and engineers studied the Shroud in great detail. All but one concluded the cloth is the burial Shroud of Christ.

The only chemical microscopist in the group came to a negative conclusion and has maintained his position in published papers and lectures since 1979. He found no blood on the shroud as reported by others in the group. He studied 32 sticky tape samples that had been applied to the Shroud surface in a variety of image areas. All 19 of these image tapes showed an abundance of tiny $<1$-micron red-orange particles. These particles were identified as red ochre pigment by size, shape, color, refractive indices and microchemical tests using polarized light microscopy, by energy-dispersive $x$ ray fluorescence (EDS) and $x$-ray diffraction. There were also tiny vermilion pigment particles in the blood image areas and these were also confirmed by all of the tests listed above for ochre. Yellowed cellulose fibers found predominantly in the image areas are typical of yellowing fibers coated with organic fluids. These image-area yellow fibers were determined to be coated with a collagen tempura by microchemical tests. This paint medium, commonly used in the 14 th century explains the yellowed linen fibers, the excellent dispersion of individual pigment particles and the difficulty experienced in picking the pigment particles from the fiber surfaces.

All of the image areas and only the image areas showed the presence of red ochre. The vermilion pigment was found only in the blood image areas. All of the results of this microscopical study consistently confirm the 1355 date and corroborate the report of Bishop Henri at the time that he knew the artist who painted the Shroud. In 1988, three laboratories obtained a date of 1325 for the origin of the linen cloth.

The mystery of the Shroud is - How could the other members of the 1978 study group have found the Shroud to be authentic? And why do they ignore the microscopical data and conclusions?

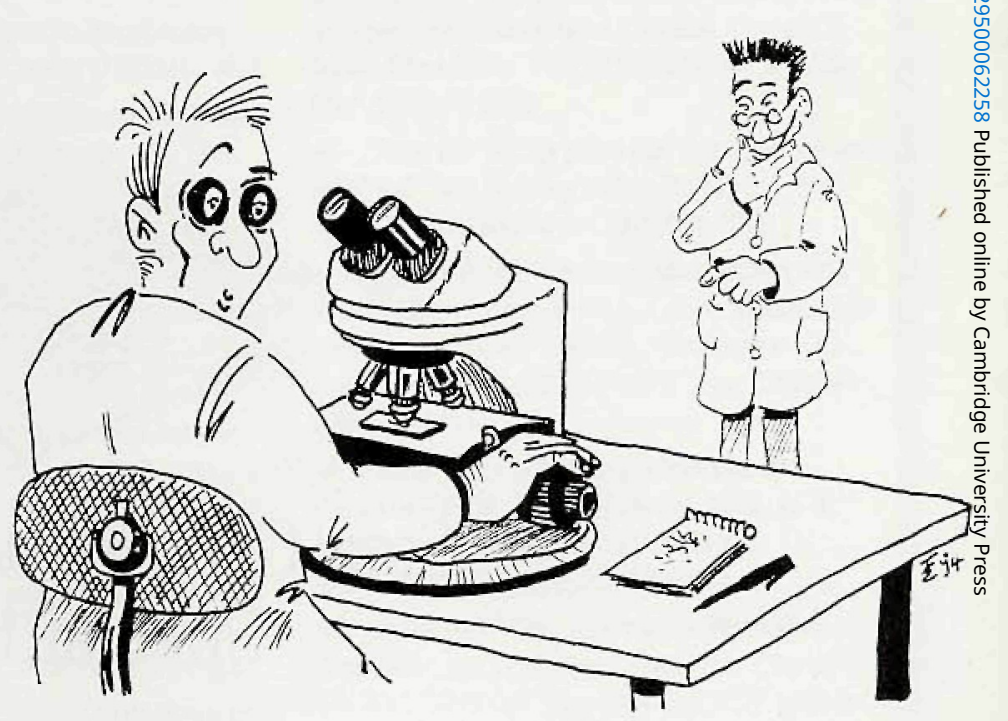

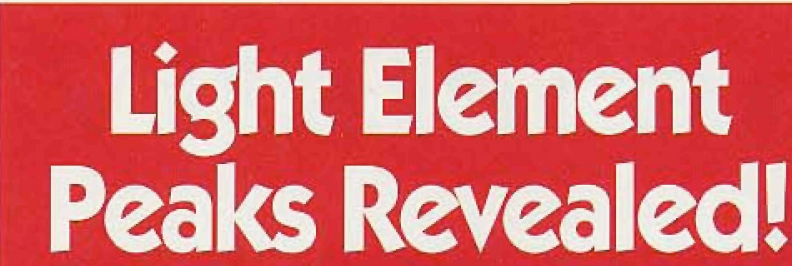

\section{Oil Film on EDX Windows Removed:}

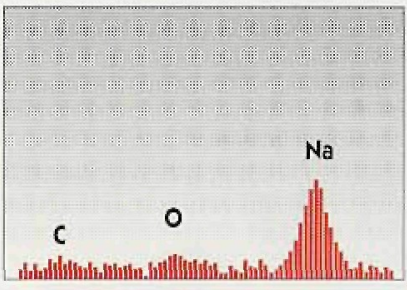

Oily Window

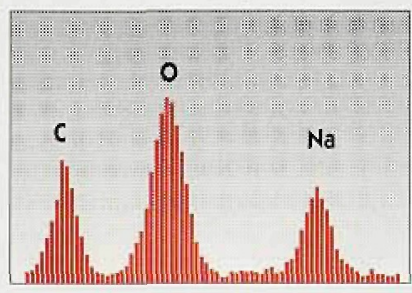

Clean Window
Oil build-up on EDX detector windows can ruin sensitivity for light element X-rays in SEMs. To stop oil condensation and keep the system clean, smart SEM users rely on the XEI Scientific SEM-CLEAN ${ }^{\top M}$ system.

Result: Consistent light element $\mathrm{X}$-ray results and contaminationfree pictures. The Nitrogen purge of the inexpensive SEM-CLEAN system actively cleans your electron microscope while you're away.

\section{SEM-CLEAN"' Stops the Oil STIEITIC}

3124 Wessex Way, Redwood City, CA 94061 415-369-0133 - Fax 415-363-1659

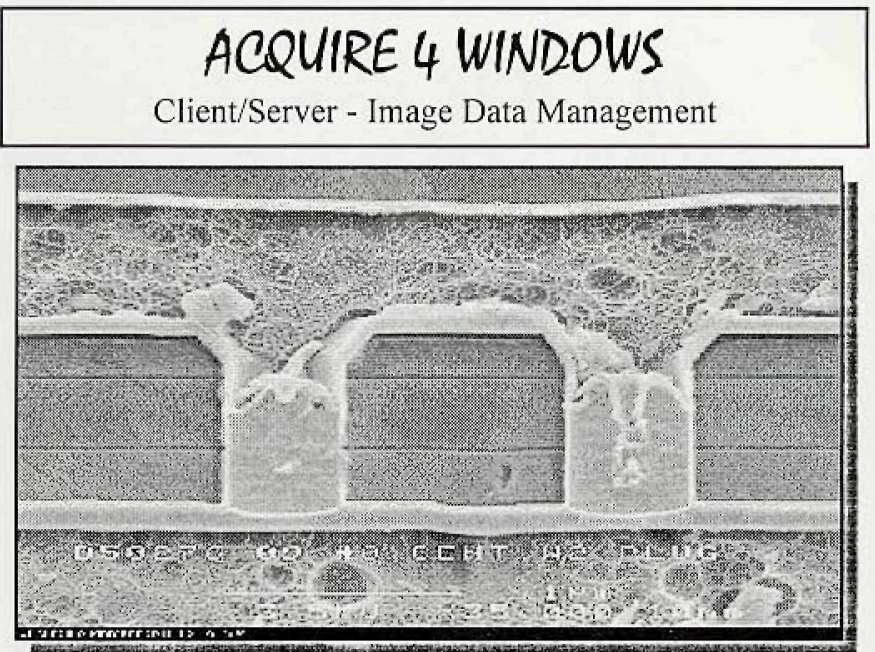

Directly plug-in your slow scan SEM images into

Windows Access and Paradox. Drag and Drop images from Acquire into popular word processors, spreadsheets.

ACQUIRE's new OLE2 features allow one to dragin-drop, import almost any type of image file into our image gallery, display single images, show all images as a slide show, preform in-place image editing.
-32Bit adjustable image compression. -Relational DataBase

-Windows OLE2 plug-in to Internet. -Windows OLE2 plug-in to Oracle. -Image text/graphics overlay. -RGB/TWAIN/Digital image capture. -Image Enhancement tools.

-Network desktop image viewer. -8 bit to 24 bit capture and display. -12 import/export image formats. -OLE2 Windows image importer. -ImagePro analytical plug-in.

-Free demo Disk-

Advanced Datalare Systems

I-800-880-8979

jhilton@rmii.com 


\section{TIMES THE X-RAY COUNT RATE ON YOUR FIELD EMISSION SEM!}
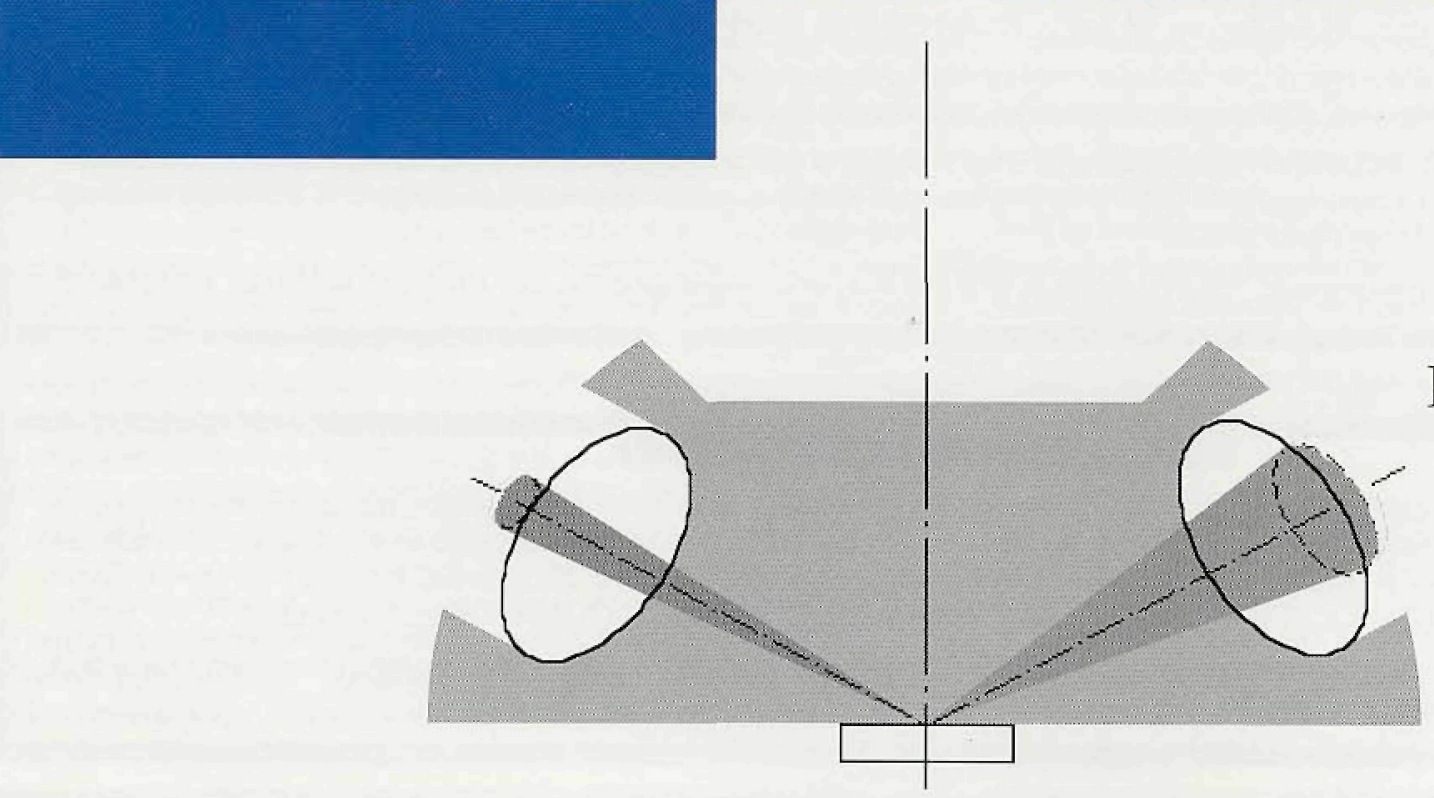

PRISM $^{60}$

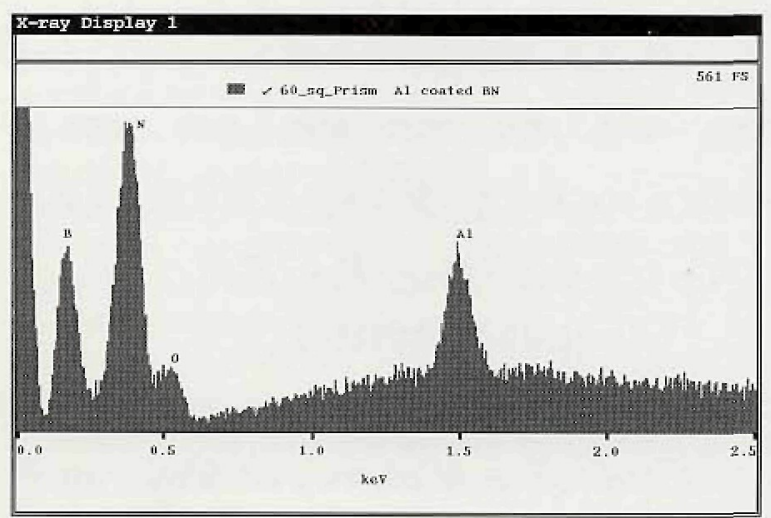

PRISM ${ }^{60}$ EDS Detector

- $60 \mathrm{~mm}^{2}$ active area $\mathrm{x}$-ray detector

- Standard size detector housing

- Excellent Light element sensitivity

- 25:1 Boron Peak/Valley ratio

- Patented Digital Pulse Processing. . . ... turns dead time into counting time!

With the PRISM ${ }^{60}$ Digital X-ray Detector you no longer have to compromise good imaging conditions to get rapid and precise $\mathrm{x}$-ray data. Six times more detection area means six times more data. . ... without sacrificing high resolution SEM operation.

Improve the speed of $x$-ray analysis on any SEM. . Call PGT today!

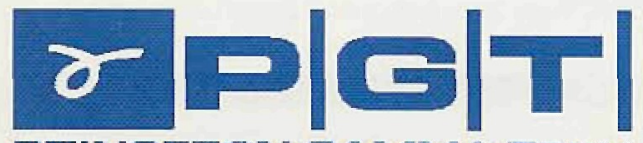

